\title{
METODOLOGIA PARA A MONITORIZAÇÃO E GESTÃo DA PESCA DE ARRASTO EM PORTUGAL. O EXEMPLO DO SIG GEOCRUST 1.0
}

\author{
JoAna Simões ${ }^{1}$ \\ Carlos Pinto ${ }^{2}$ \\ Manuel Afonso-Dias ${ }^{3}$
}

\begin{abstract}
Resumo - Este trabalho pretende dar a conhecer o Sistema de Informação Geográfica (SIG) GEOCRUST 1.0, desenvolvido por programação, no âmbito do projecto de investigação pesqueira DGXIV 99/059, Use of Satellite GPS data to map effort and landings of the Portuguese crustacean fleet (GEOCRUST), realizado na Universidade do Algarve. Esta aplicação informática, do tipo stand-alone, foi desenvolvida, fundamentalmente, com o objectivo de obter e cartografar informação de esforço e de rendimentos da pesca da frota portuguesa de arrasto de crustáceos, a partir de dados GPS de localização geográfica e da velocidade dos navios de pesca e das suas capturas. O sistema desenvolvido constitui uma proposta metodológica a adoptar em Portugal para a monitorização e gestão das pescarias de arrasto, em particular da pesca de crustáceos.
\end{abstract}

Palavras-chave: Sistemas de Informação Geográfia (SIG), programação, base de dados, GPS, pescas, litoral Sul e Sudoeste de Portugal.

\footnotetext{
Abstract - A METHODOLOGY FOR THE MONITORING AND MANAGEMENT OF TRAWL Fisheries in Portugal. The SIG eXample of GEOCRUST 1.0. This paper aims to present the Geographical Information System GEOCRUST 1.0, a computer programme developed within the fishery research project DGXIV 99/059, Use of Satellite GPS data to map effort and landings of the Portuguese crustacean fleet (GEOCRUST), in the University of Algarve. This stand-alone application was developed essentially to map fishing effort and catch per unit effort (CPUE) information for the Portuguese crustacean trawl fleet, operating off the Southwest and South coasts of Portugal. The data available for this study included GPS vessels' geographical positions and speed, transmitted via satellite to the Portuguese fisheries inspection authorities, and their catch reported to the Portuguese Directorate-General of Fisheries. GEOCRUST 1.0

1 Geógrafa, estudante de doutoramenro no Centre for Advanced Spatial Analysis, University

2 Informático, estudante de mestrado em Inteligência Artificial na Faculdade de Engenharia da Universidade do Porto e bolseiro de investigação na FCMA da Universidade do Algarve. E-mail:

3 Biólogo pesqueiro, Professor auxiliar da Universidade do Algarve. E-mail: madias @ualg.pt.
} College of London. E-mail: j.simoes@ucl.ac.uk cpinto@ualg.pt Tel: 289800 929; Fax: 289813252 
was developed in ${ }^{\circledR}$ VISUAL Basic 6.0 using two ActiveX control: ADODB, to connect with the ${ }^{\circledR}$ MsAccess 2000 database and ${ }^{\circledR}$ MAPOBJECTS 2.0 PRO, to communicate with the maps and provide GIS facilities. The application includes seven modules with the following objectives: Module 1 - to map and edit the original VMS data for a single vessel or group of vessels, for different periods of time; Module 2 - to analyse the trawl speed of each vessel; Module 3 - to define the boundaries of all fishing trips performed by vessel along the year; Module 4 - to identify and define the trawl hauls, within each fishing trip; Module 5 - to issue maps of fishing effort and CPUE; Module 6 - for exploratory data analysis and to extract data from the DB for further use in other computer packages and Module 7 - to recreate the activity of a single vessel, group of vessels or the total fleet, for a fixed period of time. This GIS application, fully developed by programming, has the advantage of being able to be used on its own without any need for external software support. The system developed is a methodological proposal to implement in Portugal, to monitor and manage the trawl fisheries, in particular, the crustacean trawl fishery.

Key words: Geographic Information System (GIS), computer programming, databases, GPS, fisheries, Southwest and South coasts of Portugal.

Resumé - Une METHOdologie déVELOPPÉE POUR LA MONITORIZATION ET GESTION des pêches au chalut au Portugal. L'exemple du GeoCrust 1.0. Ce travail a pour objectif la présentation du Système d'Information Géographique GEOCRUST 1.0, programme informatique développé à l'Université de l'Algarve, dans le cadre du projet de recherche sur la pêche DGXIV 99/059 Use of Satellite GPS data to map effort and landings of the Portuguese crustacean fleet (GEOCRUST). Cette application informatique, de type stand-alone, a été développée dans le but de cartographier les informations sur l'effort de pêche et les rendements de pêche des chalutiers à crustacés portugais, qui opèrent au large des côtes Sud et Sud-ouest du Portugal. Les informations disponibles pour cette étude incluent les captures et les données GPS des navires (localisation et vitesse), transmises par satellite aux autorités portugaises d'inspection des pêches. Le système développé propose une méthode pour le suivi informatique et la gestion des pêches au chalut, en particulier celles des crustacés, adaptable au Portugal.

Mots-clés: Système d'Information Géographique (SIG), programmation, banque de données, GPS, pêches, côtes Sud et Sud-ouest du Portugal.

\section{INTRODUÇÃO E ENQUADRAMENTO CIENTÍFICO}

GEOCRUST foi um projecto de investigação pesqueira, financiado pela Comissão Europeia (Study Contract 99/059), realizado entre Abril de 2000 e Maio de 2002 (AFonso-Dias et al., 2002). Neste projecto foram usados dados, transmitidos via satélite, de posicionamento e velocidade dos navios portugueses licenciados para a pesca de arrasto de crustáceos em Portugal. Estes dados, obtidos pelo Sistema Português de Monitorização das Capturas, denominado MONICAP, têm sido utilizados apenas para fins de fiscalização pela Inspecção Geral das Pescas (IGP) (VAsconcelos, 2000). Neste projecto, e pela primeira vez em Portugal, estes dados foram usados com fins científicos, no âmbito da investigação pesqueira. 
O objectivo principal do projecto consistiu em estimar a distribuição espacial do esforço de pesca (medido em número de lances de arrasto, número de horas de arrasto ou área arrastada) e das capturas por unidade de esforço (CPUE) - capturas por hora de arrasto - das espécies-alvo da frota de arrasto de crustáceos que actua, essencialmente, nas costas Sudoeste e Sul de Portugal (abaixo de $39^{\circ} \mathrm{N}$ ). O esforço de pesca traduz a intensidade com que os recursos pesqueiros são explorados pela frota pesqueira. A CPUE reflecte a abundância dos recursos pesqueiros, sujeitos a exploração.

A avaliação destas medidas e, não menos importante, da sua distribuição espacial, é fundamental na gestão dos recursos pesqueiros, tanto do ponto de vista da sua preservação, como do aumento da produtividade da pesca. As espécies de crustáceos alvo da pescaria de arrasto portuguesa são o lagostim (Nephrops norvegicus), a gamba (Parapenaeus longirostris) e o camarão-vermelho (Aristeus antennatus).

\section{DADOS DE BASE}

Os dados MONICAP disponíveis para este projecto, relativos aos anos de 1998 e 1999, foram disponibilizados pela IGP, enquanto a informação relativa às capturas por espécie, por embarcação e viagem de pesca, durante o mesmo período de tempo, foi cedida pela Direcção-Geral das Pescas e Agricultura (DGPA). Embora os dados MONICAP proporcionem informações precisas de localização espacial (latitude e longitude), os dados da DGPA reportam-se às capturas vendidas em lota, não havendo qualquer informação sobre a sua proveniência. Sabe-se, apenas, que as capturas foram efectuadas nas costas Sudoeste e Sul de Portugal. Em Portugal, não é autorizada a venda de pescado fora da lota e a principal lota de crustáceos situa-se no Algarve, em Vila Real de Santo António (VRSA).

Estes dois conjuntos de dados foram integrados numa única Base de Dados (BD) e o cerne deste estudo consistiu em relacionar, de uma forma sistemática, as localizações dos navios, provenientes do sistema MONICAP, com as capturas efectuadas. Como o conjunto de pontos MONICAP engloba todas as posições geográficas dos navios (em intervalos de dez minutos), foi necessário filtrar toda a informação para obter apenas a parte em que os navios estiveram efectivamente a pescar, i.e., a arrastar, considerando a arte da pesca utilizada. São apenas estes troços de informação espacial que devem ser relacionados directamente com as capturas declaradas na lota. Para tal, foi fundamental definir, dentro do conjunto de pontos MONICAP disponível para cada navio, qual a parte que correspondia a viagens de pesca, ou seja, o percurso efectuado pelo navio desde que sai do porto para pescar até regressar a esse ou a outro porto para descarregar o pescado, com vista à sua venda em lota e, dentro de cada viagem identificada, seleccionar as partes correspondentes a lanços de pesca de arrastos (os arrastos). 
Todas estas tarefas têm uma óbvia componente espacial, presente tanto ao nível da visualização da informação, como na própria construção dos resultados. De facto, pode dizer-se que o carácter espacial deste estudo se manifestou a priori, na preparação dos dados de base, a posteriori na disponibilização dos resultados e, entre estes dois momentos, ao longo de todo o projecto, no cruzamento de informação, intervindo mesmo nos próprios algoritmos criados. Por este motivo, tornava-se fundamental o desenvolvimento de um Sistema de Informação Geográfica (SIG), que integrasse os dados MONICAP com os dados de capturas, numa plataforma espacial.

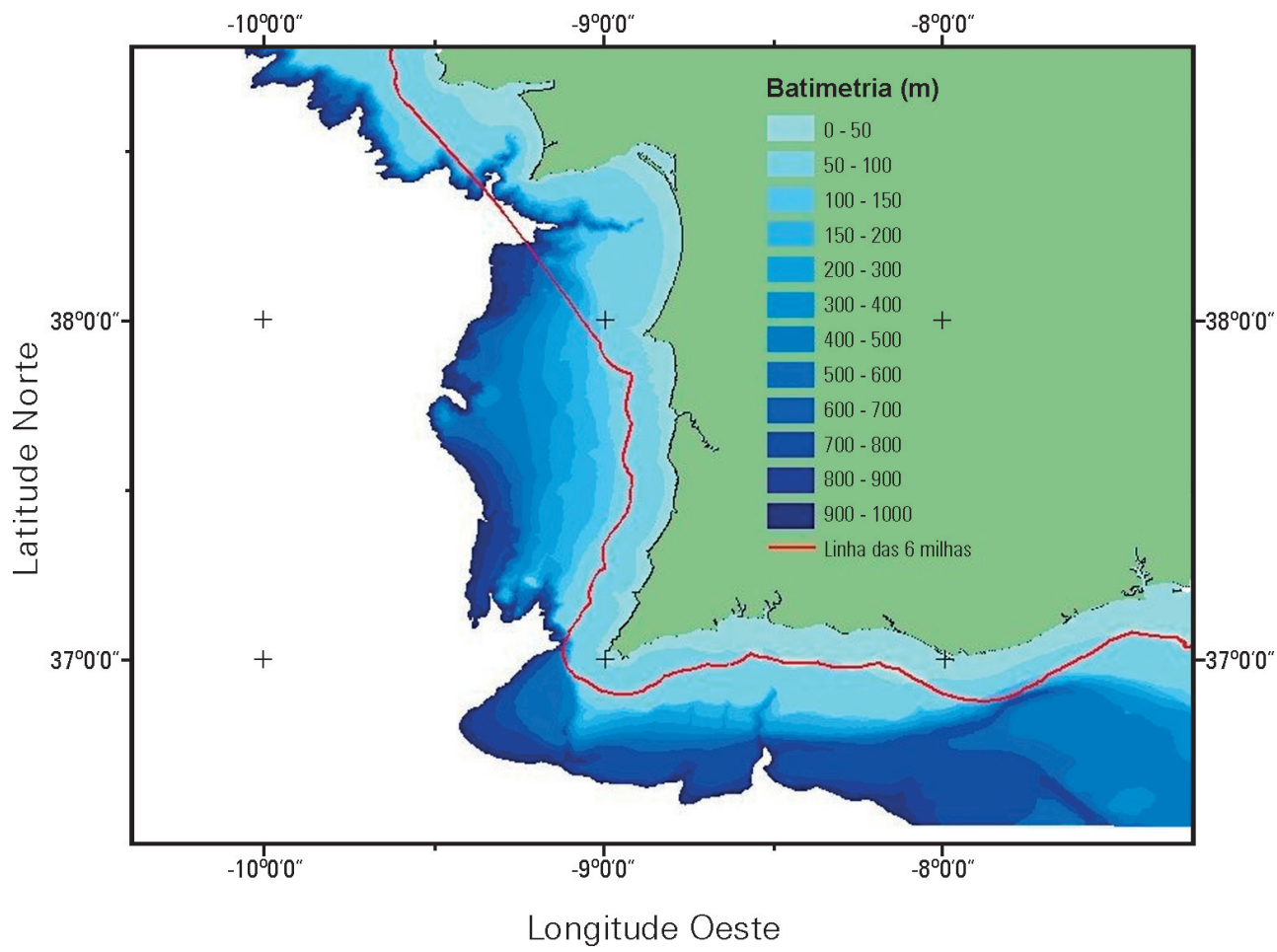

Fig. 1 - GEOCRUST 1.0 - Diferentes coberturas da carta digital geo-referenciada: linha de costa, batimetria, distância à linha de costa dentro da qual não é autorizada a pesca de arrasto em Portugal (Portaria N. ${ }^{\circ} 1102-E / 2000$ de 22 de Novembro). De notar que, entre os cabos Raso, Espichel e Sines, esta distância é medida a partir das respectivas linhas de base recta.

Fig. 1 - GEOCRUST 1.0 software: Different layers of information are shown: coast line, depth contours, and distance from the coast (red line) inside which, in accordance with Regulation No. 1102-E/2000 of November $22^{\text {nd }}$, trawl fishing is not allowed in Portugal (it is worth noting that between Cape Raso and Cape Espichel and between Cape Espichel and Sines, this distance is measured from a straight line joining these three capes/locations two by two). 
$\mathrm{O}$ enquadramento espacial para a informação da BD consistiu num conjunto de cartas digitais com informação sobre a zona de estudo, nomeadamente a linha de costa, alguma toponímia (nome dos portos de pesca frequentados pelos navios de arrasto de crustáceos) e a classificação do tipo de sedimentos do fundo do mar, a batimetria e a linha das seis milhas ${ }^{4}$ (fig. 1).

Estas cartas, que cobrem a costa Sudoeste e Sul de Portugal, abaixo dos $39^{\circ} \mathrm{N}$, foram preparadas compilando informação das cartas de pesca disponíveis: AP5, AP6, AP7 e AP8, publicadas pelo Instituto Hidrográfico (IH), à escala 1:150 000. As cartas foram geo-referenciadas e vectorizadas por forma a criar os ficheiros de entrada no SIG.

\section{RESOLUÇÃO DE PROBLEMAS TÉCNICOS}

O software utilizado no início do projecto (Abril de 2000) foi o ${ }^{\circledR}$ Microsoft Access, no qual foi implementada a BD deste estudo, e o ${ }^{\circledR}$ ArCVIEW 3.2 da ESRI. É de salientar que, no início deste trabalho, não se encontrava ainda disponível o software ${ }^{\circledR}$ ARCGIS 8.1, com que se poderia ter superado muitas das dificuldades encontradas, e que chegou a ser adoptado na execução de algumas tarefas pontuais, na fase final deste estudo. $O{ }^{\circledR}$ ARCVIEW 3.2, e mesmo a versão corrigida 3.2a, possui muitas limitações relativamente às necessidades deste projecto, nomeadamente no que diz respeito à sua relação com BD externas. Na realidade, os mecanismos que possui de ligação com as BD permitem inquirições (queries), mas estas traduzem-se em tabelas temporárias dentro do próprio ${ }^{\circledR}$ ARCVIEW, que não são actualizáveis, nem editáveis.

Devido à natureza deste estudo, era fundamental que houvesse bi-direccionalidade entre a BD e a interface espacial, permitindo que as alterações efectuadas num e noutro meio estivessem imediatamente disponíveis nos dois ambientes. Uma solução que poderia, pelo menos parcialmente, resolver este problema seria converter a BD para DBASE, uma vez que os ficheiros de extensão .dbf são os controladores (drivers) nativos do ${ }^{\circledR}$ ARCviEw e, ao contrário do que sucede com os ficheiros . $m d b$, do ${ }^{\circledR}$ Microsoft Access, é possível editá-los dentro deste programa. Outra alternativa, mais poderosa, apontada pela própria ESRI, seria a criação de um tema cartográfico (layer) de acesso entre a BD e o ${ }^{\circledR}$ ARcview: o ARcSDE. A instalação deste sistema, para além de dispendiosa, pressupunha ainda a conversão da BD para ORACLE ou SQL.

No entanto, nenhum destes procedimentos ultrapassaria o fraco desempenho do ${ }^{\circledR}$ ARCVIEW ao lidar com um volume de informação, no limiar dos limites das suas capacidades. De facto, a BD possui um grande volume de dados (cerca de 30 tabelas), chegando a ter tabelas com mais de 1 milhão de registos. Estas

4 A frota portuguesa está legalmente proíbida de pescar a menos de seis milhas da linha de costa pelo que esta linha é utilizada para fins de fiscalização. De notar que, entre os cabos Raso, Espichel e Sines, esta distância é medida a partir das respectivas linhas de base recta (ver Regulamento da Pesca por Arte de Arrasto - Portaria n. ${ }^{\circ}$ 1102-E/2000 de 22de Novembro). 
debilidades do software eram visíveis, quando o computador bloqueava numa simples visualização de uma inquirição à $\mathrm{BD}$.

Por esta razão, mas também pela motivação das enormes vantagens que um software stand-alone traz, foi desenvolvida uma aplicação de raíz. Esta aplicação, denominada GEOCRUST 1.0, foi integralmente implementada em ${ }^{\circledR}$ VISUAL BASIc 6.0, com a inclusão de diversos controlos ActiveX, de entre os quais há a salientar o ADODB e о ${ }^{\circledR}$ MAP OBJECTS PRO 2.0, da ESRI, pela relevância das funcionalidades que providenciaram. O ADODB foi o controlo que permitiu a ligação com a $\mathrm{BD}$, utilizando um jet engine do Windows: através de strings de SQL e de recordsets foi possível alterar e visualizar tabelas de ${ }^{\circledR}$ Microsoft Access dentro do GEOCRUST 1.0. O ${ }^{\circledR}$ MAP OBJECTS ${ }^{5}$ foi o controlo que providenciou as funcionalidades de SIG presentes no GEOCRUST 1.0. De facto, utilizando este controlo, é possível 'recriar', através de programação, todas as funcionalidades presentes no ${ }^{\circledR}$ ARCVIEW e ainda algumas presentes no ${ }^{\circledR}$ ARcINFO. Incluem-se nestas funcionalidades a entrada (input) e saída (output) de informação geográfica e ainda funções de análise espacial.

A aplicação GEOCRUST 1.0 foi desenvolvida em módulos, cada um deles com uma determinada função. Cada um dos módulos pode ser usado separadamente, o que não seria possível, caso o estudo tivesse sido desenvolvido num software SIG comercial. A aplicação está também preparada para aceitar séries temporais de dados, para além dos dados utilizados no seu desenvolvimento, e encontra-se suficientemente flexível para que, com pequenas alterações na programação, possam ser facilmente incorporadas outras áreas geográficas e analisados dados de outras pescarias de arrasto.

\section{DESCRIÇÃO DA APLICAÇÃO}

O software GEOCRUST 1.0 é constituído por um menu principal, onde se selecciona a BD do projecto e por sete módulos, a que se pode aceder através deste menu. Também é possível, dentro do menu principal, aceder a um conjunto de rotinas, que efectuam operações gerais como actualizações da BD ou criação de ficheiros com formato shapefile.

Em seguida, será feita uma breve descrição de cada um destes módulos, salientando-se as funções consideradas mais relevantes.

\section{Módulo Análise Preliminar}

Este módulo foi desenvolvido essencialmente com o objectivo de proporcionar uma avaliação da qualidade dos dados MONICAP. Ao iniciar este estudo, houve a necessidade de criar uma aplicação que acedesse à informação espacial presente na base de dados e a apresentasse visualmente de forma perceptível. Uma vez que se trata de um conjunto muito grande de dados, mesmo conside-

\footnotetext{
5 http://www.esri.com/software/mapobjects/index.html (último acesso em Outubro de 2003).
} 
rando os navios individualmente, houve necessidade de efectuar algum tipo de separação temporal. Assim, não havendo ainda um conhecimento que permitisse a separação em viagens de pesca, nos termos em que mais tarde foram definidas, foi considerado um critério simples e expedito para a segmentação dos dados de cada navio em viagens ad-hoc, considerando o fim de uma viagem de pesca, e o início da viagem seguinte, às 4 horas da madrugada do dia em que se registava uma venda em lota. Este critério teve como base informação sobre o modo de funcionamento da pesca de arrasto de crustáceos no Algarve (horas de regresso e de saída para o mar dos arrastões e altura em que se processa a venda de crustáceos na lota de VRSA).

Este critério, completamente automático, criou diversos problemas como o de partir 'verdadeiras' viagens de pesca, mas cumpriu os objectivos iniciais de partição dos dados para possibilitar a sua Análise Preliminar. A visualização proporcionada por este módulo pode ser feita para um navio seleccionado, para uma ou mais viagens e para um ou mais trimestres. Um template apresenta, automaticamente, o padrão espacial da actividade de cada navio, por trimestre, num determinado ano.

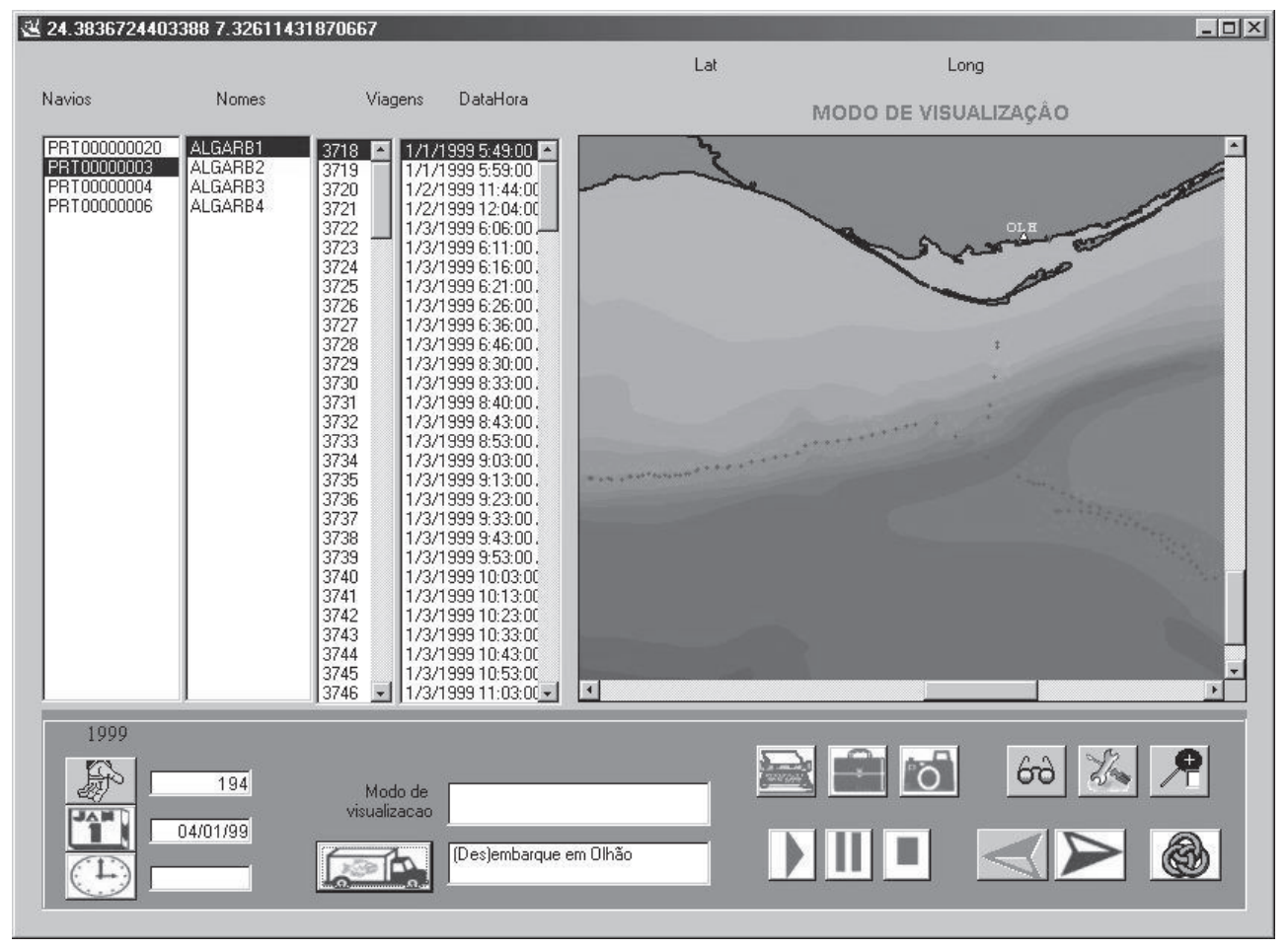

Fig. 2 - GeOCRUST 1.0 - Módulo Análise Preliminar: modo de visualização de dados de localização geográfica de navios de pesca de arrasto de crustáceos.

Fig. 2 - GEOCRUST 1.0 software - Module Preliminary Analysis: tools for preliminary analyses by plotting the spatial distribution of a particular crustacean fishing trawler. 
Durante a preparação da BD, verificou-se que os dados MONICAP tinham um grande número de erros (localizações inconsistentes) que necessitavam de correcção. Assim, dentro deste módulo, é possível fazer a detecção de outliers (pontos com localizações díspares) e pontos com a mesma data e hora, para a mesma ou diferentes localizações, e apagar ou corrigir estes pontos (fig. 2). Em qualquer dos casos mencionados é preciso efectuar operações de leitura, inserção ou remoção de registos na BD. Os valores alterados ou removidos são guardados em tabelas apropriadas, criadas para o efeito.

\section{Módulo Análise de Frequências de Velocidade}

Este módulo permite analisar a distribuição de Frequências de Velocidade de um navio, num dado período de tempo, e comparar as distribuições de frequências de velocidade de vários navios, ou de um mesmo navio, em períodos de tempo diferentes. A distribuição de frequências de velocidade apresenta tipicamente duas modas, uma principal, de velocidades baixas (entre dois e três e meio nós ${ }^{6}$ ) e uma secundária, de velocidades elevadas (entre oito e dez nós). A primeira moda corresponde, essencialmente, à velocidade de arrasto, e a segunda, à navegação de cruzeiro. Foram identificadas diferenças na velocidade de arrasto entre navios e, em alguns casos, para o mesmo navio, em períodos de tempo diferentes. Esta constatação impediu que se pudesse seleccionar, de entre os dados MONICAP, por simples filtragem, a informação relativa aos períodos de arrasto.

\section{Módulo Marcação de Viagens}

Este módulo foi criado como uma ferramenta para definir os verdadeiros limites (início e fim) de cada viagem de pesca, para cada navio seleccionado. O ecrã principal é constituído por um mapa mostrando a localização espacial dos pontos e um gráfico com o perfil de velocidades correspondente à mesma série temporal (fig. 3). Desta forma, os arrastos (efectuados a uma velocidade reduzida e constante) tornam-se facilmente reconhecíveis. Nalguns casos, em que os registos de velocidade apresentavam anomalias sistemáticas, mas as posições geográficas se encontravam correctas, calculou-se a velocidade média entre duas posições geográficas consecutivas.

Os limites das viagens foram definidos utilizando procedimentos automáticos ou semi-automáticos, conforme a qualidade dos dados. Ambos os procedimentos utilizam algoritmos em que se considera a primeira visita ao porto, imediatamente anterior a uma venda, como o fim de uma viagem e a partida, imediatamente posterior, como o início da viagem seguinte. Considerou-se que as vendas em lota, representadas na parte superior do gráfico de velocidades através de triângulos (fig. 3), eram efectuadas às 6 da manhã.

6 Nó=milhas náuticas por hora $(\mathrm{mn} / \mathrm{h})(1$ milha náutica=1852 metros). 


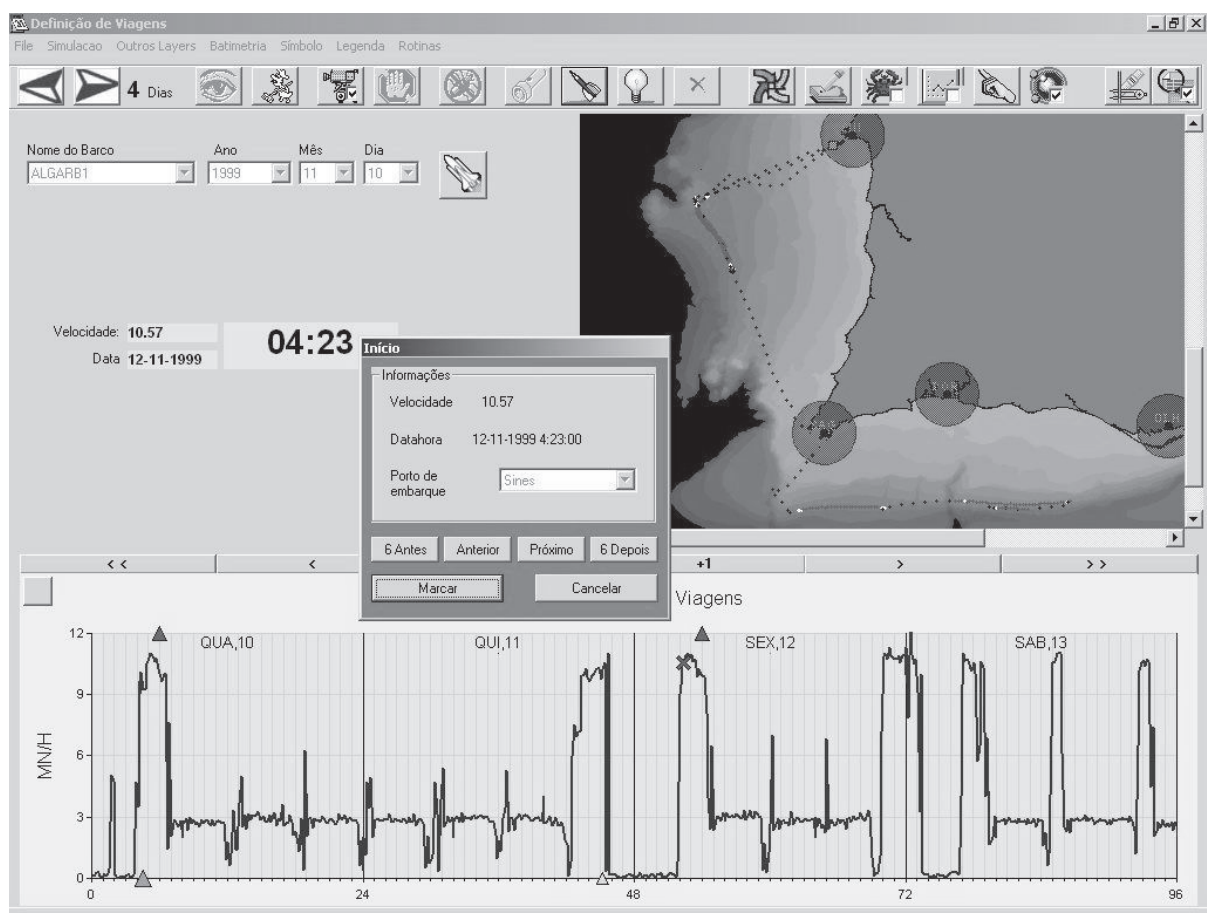

Fig. 3 - GEOCRUST 1.0 - Módulo Marcação de Viagens: marcação do início de uma viagem de pesca através de um processo semi-automático.

Fig. 3 -GEoCRUST 1.0 software - Module Fishing Trips Definition: screen display showing the definition, in a semi-automatic way, of the beginning of a fishing trip.

Estes algoritmos consideram ainda a distância do navio relativamente ao porto. Para isso, foram criadas áreas de influência (buffers) em torno de cada porto, considerando-se um círculo de raio fixo. De cada vez que um navio entra na área de influência de um determinado porto e é registada, horas depois, a venda de pescado desse navio, considera-se que o mesmo terminou uma viagem de pesca. A próxima saída da área de influência determina o início de uma nova viagem desse navio. De notar que, apesar de todos os pontos serem exibidos nos mapas, estes têm uma ordem cronológica (data e hora).

As viagens marcadas são classificadas como 'válidas', quando não há falhas de dados, ou 'parcialmente válidas', quando é possível definir os seus limites, mas não é viável determinar os limites de um ou mais arrastos devido à ausência de parte dos dados. A informação relativa ao início e fim da viagem e a classificação qualitativa da mesma são inseridos na BD em tabela apropriada.

Utilizando as funcionalidades SIG do ${ }^{\circledR}$ МАРОВJестs foi criado, neste módulo, um conjunto de ferramentas de visualização para analisar, em pormenor, a distribuição espacial dos dados (zoom e pan), para auxiliar o utilizador na marcação semi-automática dos limites das viagens. 


\section{Módulo Marcação de Arrastos}

Este módulo tem como principal objectivo a marcação dos limites (início e fim) dos arrastos efectuados dentro das viagens de pesca definidas no módulo anterior. O ecrã principal da aplicação é composto de dois mapas, um para enquadramento e outro para zoom, e um gráfico com o espectro de velocidades (fig. 4). Tal como sucedia no módulo de Marcação de Viagens, existe sincronia entre o gráfico de velocidades e, neste caso, os dois mapas.

Ao contrário do que sucedeu no módulo de Marcação de Viagens, não foi possível desenvolver um algoritmo que assegurasse uma elevada taxa de sucesso na marcação dos limites dos arrastos, através de marcação automática, para toda a base de dados. A natureza desta informação não permitiu acertar, para já, um método expedito e simples de Marcação de Arrastos sem a intervenção do utilizador em numerosos casos e, por isso, foi desenvolvido apenas um procedimento semi-automático, que requer sempre a confirmação do operador (fig. 4).

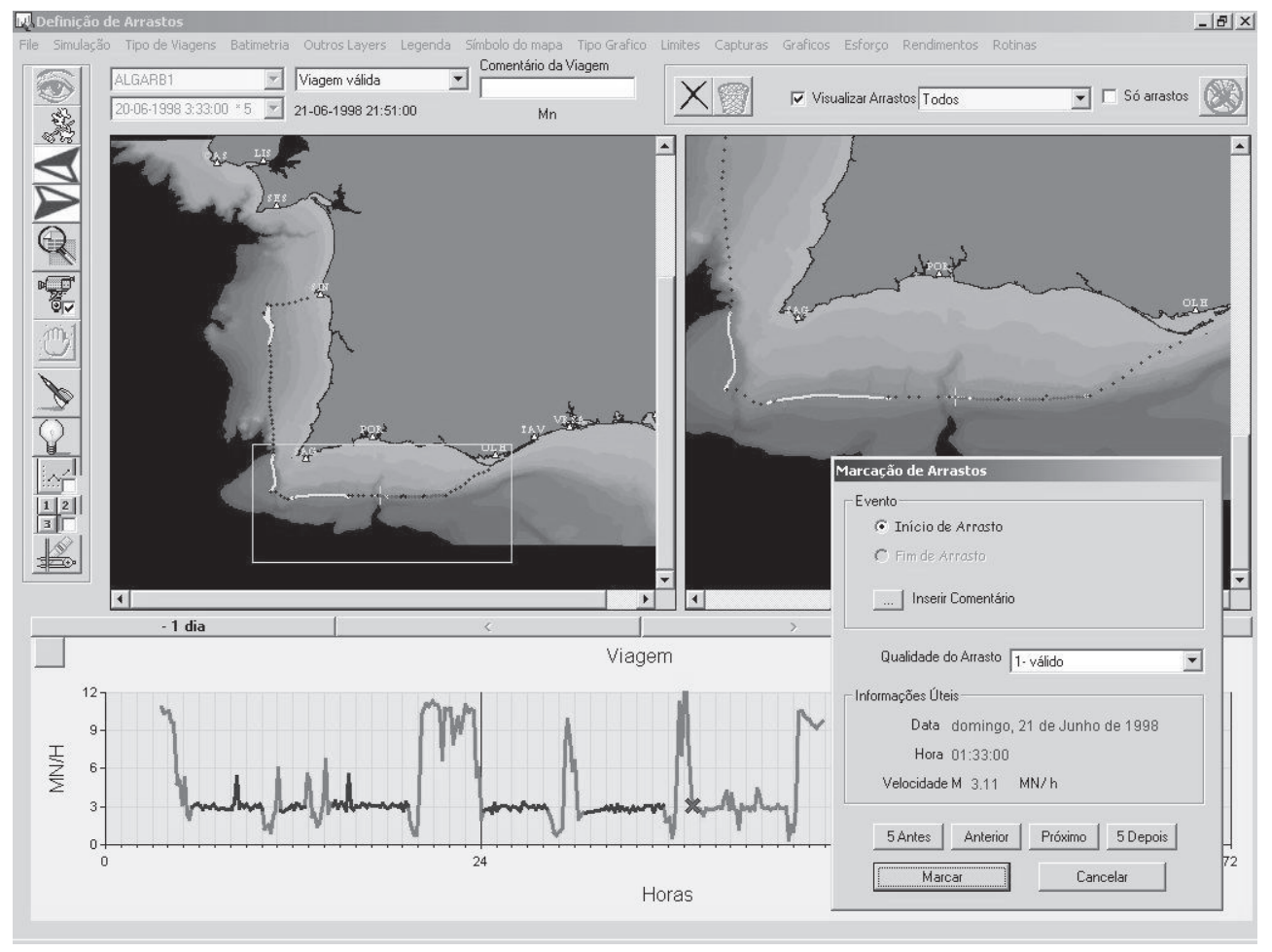

Fig. 4 - GEOCRUST 1.0 - Módulo Marcação de Arrastos: marcação do início do sexto arrasto de uma viagem de pesca para um navio seleccionado.

Fig. 4 -GEOCRUST 1.0 software - Module Trawl Hauls Definition: screen display showing the definition, in a semi-automatic way, of the beginning of the sixth trawl haul of a fishing trip from a selected vessel. 
Através da análise do espectro de velocidade mensal de cada navio foram definidos limites inferiores e superiores de velocidade de arrasto (valores correspondentes a um corte ao nível dos $4 \%$ na parte da distribuição de frequências relativa à actividade de arrasto). Esta informação foi utilizada no algoritmo de identificação dos limites dos arrastos, juntamente com um padrão de comportamentos identificados nos navios durante as operações de pesca de arrasto - manutenção de uma velocidade aproximadamente constante e variações acentuadas de velocidade no início e no fim dos arrastos (fig. 5).

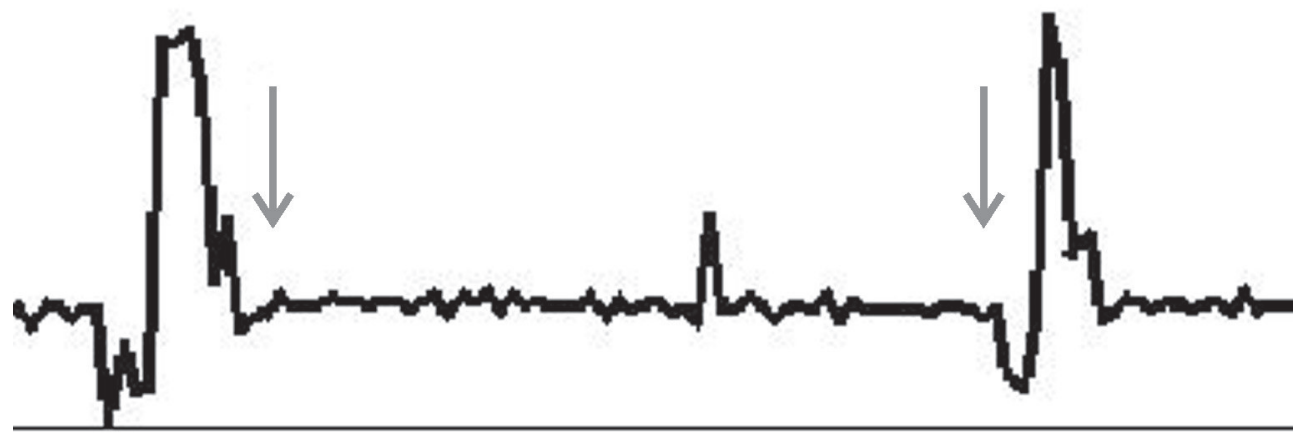

Fig. 5 - Perfil típico da variação da velocidade de um arrastão, ao longo do tempo, com o início e o fim de um arrasto assinalados com setas.

Fig. 5 - Trawler speed variation showing the beginning and the end of the trawl haul indicated with an arrow.

Através de rotinas próprias, este módulo calcula e insere na BD informação relativa à duração, distância, área arrastada (considerando uma abertura horizontal da rede de arrasto constante), localização em termos de zona geográfica (são consideradas três regiões: Lisboa, Alentejo e Algarve), nome do pesqueiro e estrato batimétrico onde se efectuou o arrasto. Para cada viagem analisada é possível visualizar, no ecrã (em tabela e sectograma), a composição específica das capturas (quantidades vendidas em lota, em quilograma, por espécie), o esforço de pesca e os rendimentos de pesca (CPUE), resultantes da divisão das capturas pelo esforço de pesca.

Só foi possível a identificação do pesqueiro em que se efectuou cada um dos arrastos após estes terem sido definidos, o que ocorreu na parte terminal do projecto GEOCRUST, após ter sido processada toda a informação disponível para este estudo. Utilizando o ${ }^{\circledR}$ ARCGIS 8.1 (e a ferramenta Geoprocessing wizard), foram construídas áreas de influência 'apertadas' em torno de todos os arrastos identificados (cerca de 15 mil) criando-se, assim, um mapa dos pesqueiros de crustáceos capturados por arrasto em Portugal, o qual constitui agora um dos temas cartográficos integrados no software (fig. 6). 


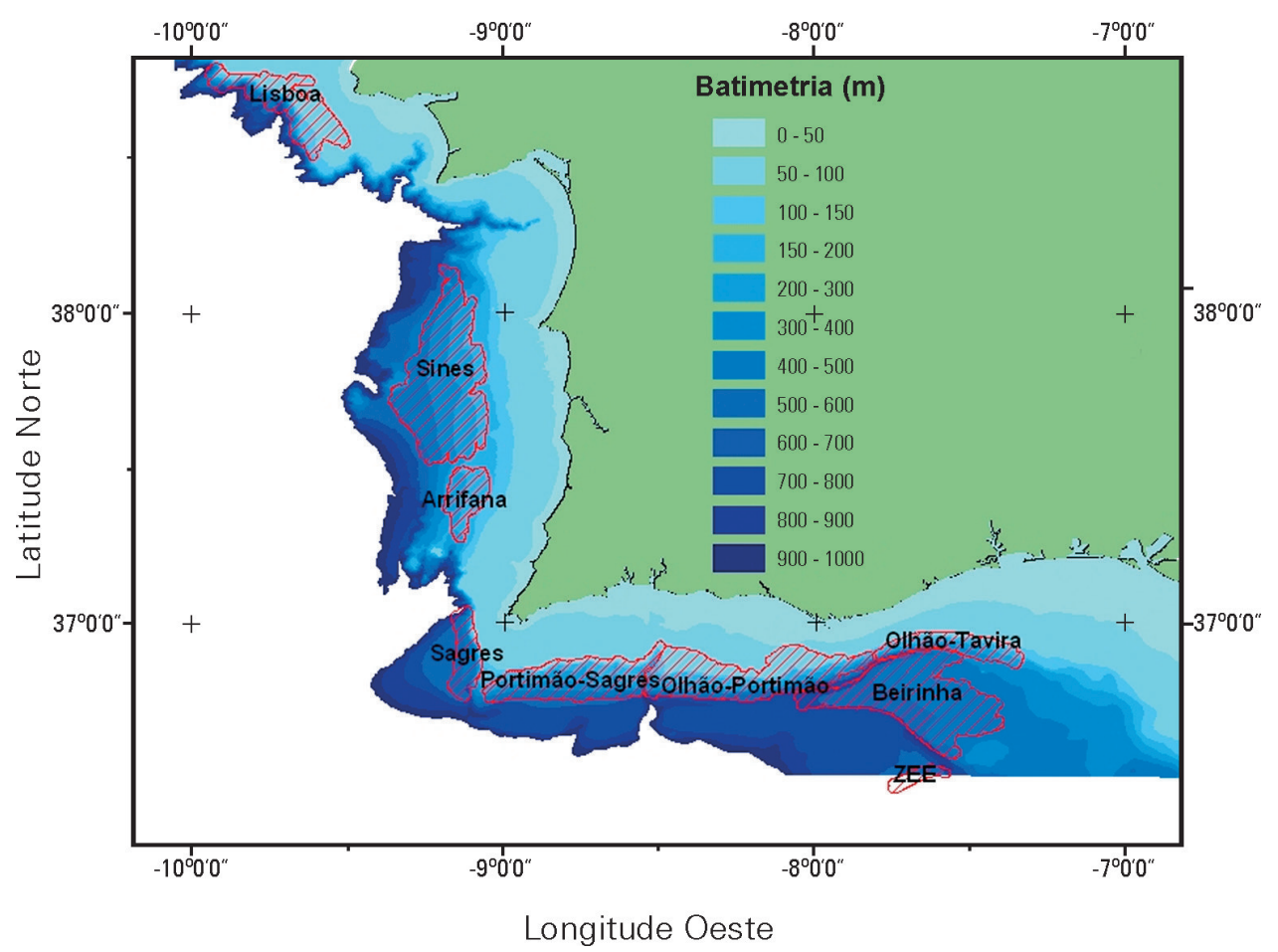

Fig. 6 - Identificação dos oito pesqueiros de crustáceos definidos no projecto GEOCRUST, ao largo das costas Sudoeste e Sul de Portugal (a Sul de $39^{\circ} \mathrm{N}$ ).

Fig. 6 - The eight crustacean fishing grounds identified off the Southwest and South coasts of Portugal (south of $39^{\circ} \mathrm{N}$ ) in the GEOCRUST project.

\section{Módulo Cartografia}

Este módulo destina-se à representação e análise da informação resultante do trabalho desenvolvido com os módulos de Marcação de Viagens e de Marcação de Arrastos. Através de menus, é possível aceder à BD e elaborar, de forma simples, uma série de consultas, seleccionando uma embarcação ou um conjunto de embarcações (a frota toda), escolhendo um determinado período de tempo (personalizado ou a definir) e uma área geográfica (toda ou apenas uma ou duas das três regióes consideradas) ou ainda um pesqueiro.

Na cartografia do esforço de pesca foram utilizados dois tipos de representação distintos. O primeiro apresenta os lances de arrasto de forma linear, permitindo ao utilizador sobrepor o resultado de várias inquirições, usando diferentes cores. Contudo, neste tipo de representação, não são visíveis as diferenças espaciais em termos de intensidade de arrasto, uma vez que existe muita sobreposição de informação devido ao facto de os navios arrastarem muito frequentemente nas mesmas áreas. Por esta razão, foi considerado um segundo 
tipo de representação: uma grelha de densidades com uma classificação baseada no número máximo de pontos por quadrícula (fig. 7). Estão disponíveis duas grelhas, uma com quadrículas de $5 \times 5$ milhas náuticas $(\mathrm{mn})$ e outra, de maior utilidade, com quadrículas de $1 \times 1 \mathrm{mn}$. O algoritmo que suporta esta função traduza inquirição criada pelo utilizador num filtro sobre o ficheiro de formato shapefile dos arrastos ao qual é sobreposta um outro com uma grelha uniforme. Depois, através de métodos do MАРОвлестs, é contado o número de pontos em cada célula e é criada a classificação dinâmica que vai atribuir cores a cada uma das células, proporcionando uma fácil percepção visual da intensidade do esforço de pesca.

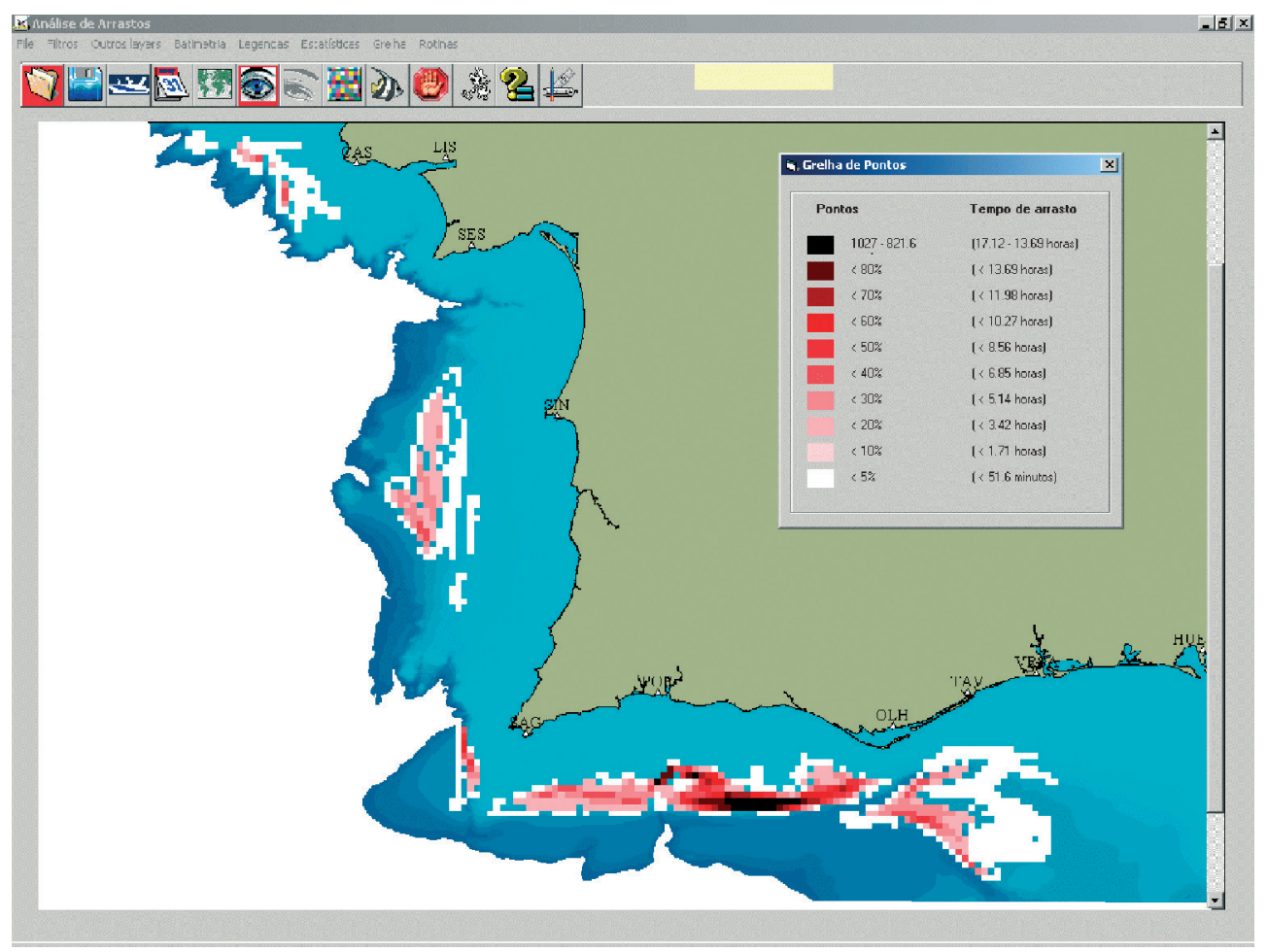

Fig. 7 - Módulo Cartografia da aplicação GEOCRUST: representação de densidades de arrasto em grelha de $1 \times 1 \mathrm{mn}$, para um determinado número de navios seleccionados, num certo período de tempo.

Fig. 7 - Module Cartography of GEOCRUST 1.0 software: grid of densities of the trawl hauls conducted by a selected number of vessels for a selected period of time.

Este módulo possibilita também o cálculo e representação cartográfica, através de sectogramas (fig. 8) ou histogramas, da CPUE para uma embarcação ou conjunto de embarcações, num período de tempo definido pelo utilizador. 


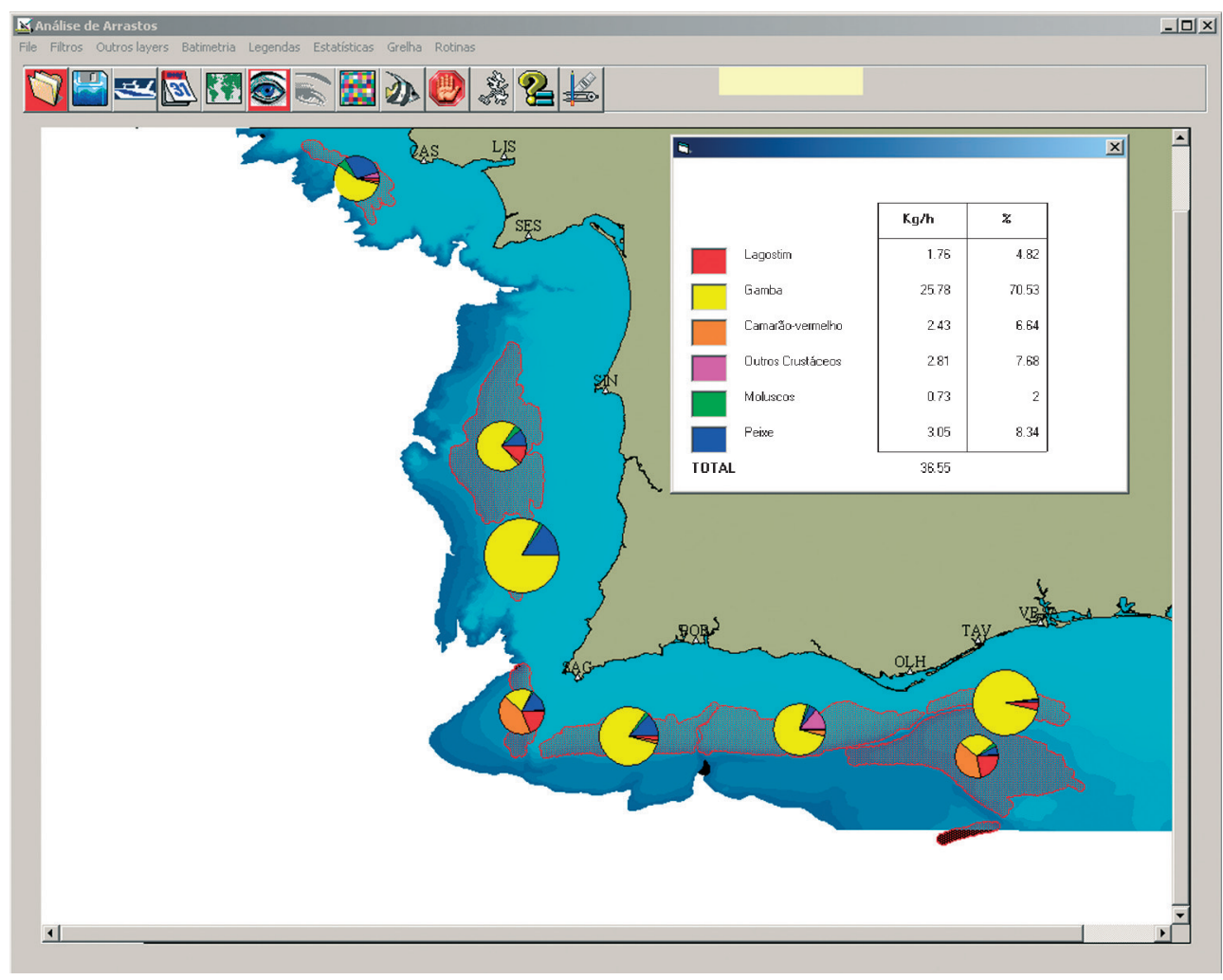

Fig. 8 - Módulo Cartografia da aplicação GEOCRUST. Sectogramas representanto as Capturas por Unidade de Esforço $(\mathrm{kg} / \mathrm{h})$, por pesqueiro, das espécies-alvo: lagostim (Nephrops norvegicus), gamba (Parapenaeus longirostris) camarão-vermelho (Aristeus antennatus), bem como dos principais grupos de espécies capturadas - peixes, moluscos e outros crustáceos.

Fig. 8 - Module Cartography of GEOCRUST 1.0 software. Pie charts of Catch per Unit Effort $(\mathrm{kg} / \mathrm{h})$, by fishing ground, of the crustacean target species: norway lobster (Nephrops norvegicus), deepwater rose shrimp (Parapenaeus longirostris) and red shrimp (Aristeus antennatus); finfish, molluscs and other crustaceans are also shown.

Muitas vezes, os arrastos correspondentes a uma viagem foram efectuados em diferentes regiões e, ainda mais frequentemente, em diferentes pesqueiros, o que torna difícil atribuir as capturas a uma determinada zona. Por esta razão, foram desenvolvidas ferramentas para seleccionar viagens exclusivas de regiões ou pesqueiros, ou seja, viagens cujos arrastos foram todos realizados apenas numa região ou num pesqueiro. A análise destas viagens que, como se veio a verificar, correspondem a uma parte significativa do total, revelou-se fundamental neste estudo. 


\section{Módulo Estatísticas}

Este módulo destina-se essencialmente à realização de algumas análises exploratórias da informação contida na BD e à extracção de informação para análise com o auxílio de outro software (análises estatísticas). Através de menus, o utilizador pode, de uma forma simples, comparar capturas, esforços de pesca e CPUE entre navios ou grupos de navios, num determinado período de tempo (mês, trimestre, semestre ou ano). Através deste módulo pode ser extraída informação de esforço de pesca, capturas e CPUE para uma determinada região ou pesqueiro, por navio ou grupo de navios. Dentro deste módulo é também possível efectuar a cartografia do esforço de pesca para as viagens seleccionadas.

\section{Módulo Recriação da Actividade da Frota}

Este módulo é uma aplicação complementar que proporciona uma recriação da actividade de pesca de um conjunto de navios seleccionados, para um determinado período de tempo, em que o utilizador controla a velocidade da recriação (fig. 9).

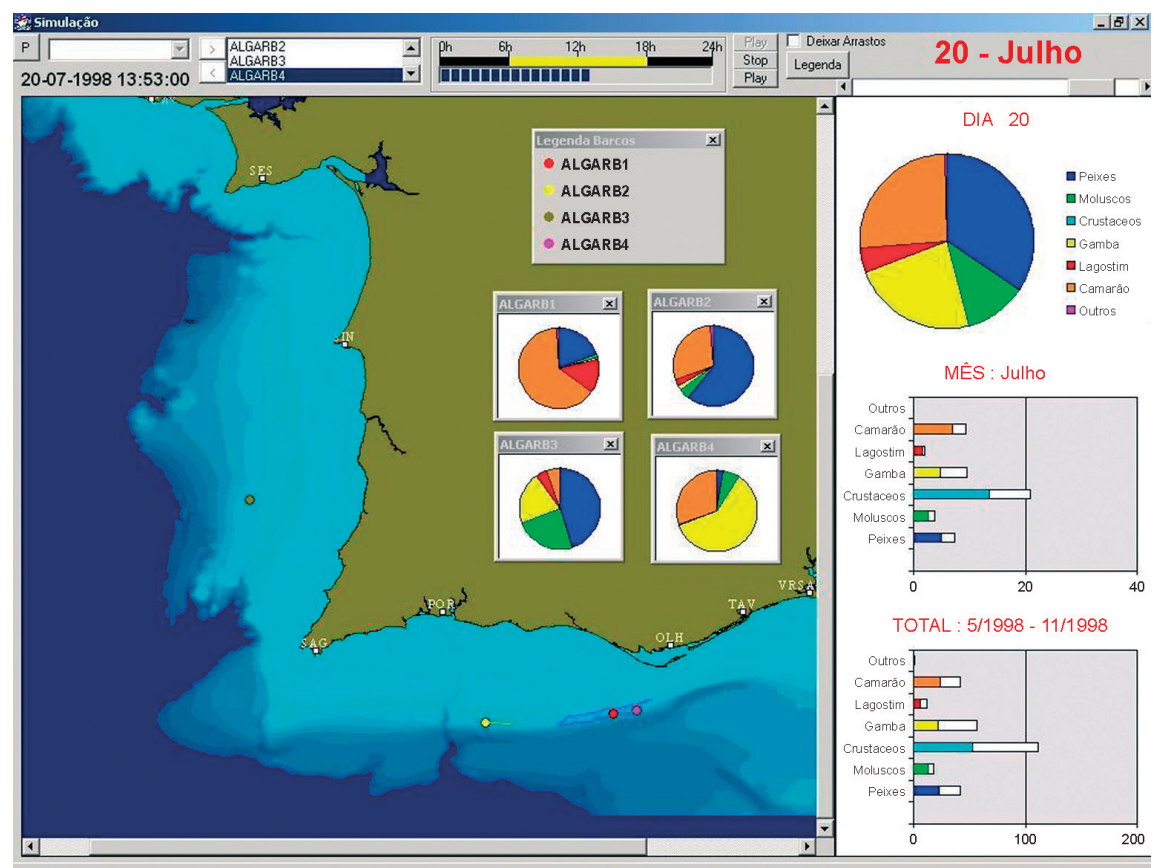

Fig. 9 - GeOCRUST 1.0. - Módulo Recriação da Actividade da Frota: simulação da actividade de quatro navios (círculos coloridos no mar) da frota de arrasto de crustáceos entre Maio e Novembro de 1998.

Fig. 9 - GEOCRUST 1.0. - Module Recreation of the fleet operations: simulation of the operations of four selected crustaceans trawlers (coloured dots inthe sea), between May and November 1998. 
Durante a execução da recriação, as capturas diárias (sectograma) e acumuladas (mensalmente e no total do período escolhido) (gráficos de barras) vão sendo mostradas automaticamente para o conjunto dos navios (fig. 9). As capturas diárias de cada um dos navios podem ser visualizadas individualmente, caso se pretenda, através de sectogramas. Embora este módulo tenha sido desenvolvido, essencialmente, para demonstrações, o mesmo revelou-se importante para a compreensão de alguns aspectos da dinâmica da frota de crustáceos, pois reproduz o seu comportamento.

\section{RESULTADOS}

O software GEOCRUST 1.0, desenvolvido no âmbito do projecto GEOCRUST, permitiu obter informação geo-referenciada de cerca de $41 \%$ do total das viagens de pesca estimadas para a frota de portuguesa de crustáceos, no período 1998/1999. Entre os principais resultados científicos deste estudo, contidos no relatório final do projecto (Afonso-Dias et al., 2002), salienta-se a definição dos contornos geográficos dos pesqueiros de crustáceos explorados por arrasto, em 1998 e 1999, e a sua caracterização, em termos de área e batimetria, e a cartografia e quantificação geográfica do esforço de pesca, capturas e rendimentos da frota de arrasto de crustáceos em 1998 e 1999.

Verificou-se que a actividade pesqueira dos arrastões da frota de crustáceos, em 1998 e 1999, foi dirigida, essencialmente, à gamba e foi realizada no Algarve, com particular incidência em dois pesqueiros: Portimão-Sagres e Olhão-Portimão. A extraordinária abundância de gamba, observada no segundo semestre de $1998 \mathrm{e}$ em 1999, parece explicar a distribuição geográfica do esforço de pesca observada.

As CPUE de cada espécie-alvo variaram ao longo dos meses e entre pesqueiros e regiões. A variação trimestral da CPUE das três espécies-alvo, por pesqueiro e, também, por região, mostrou padrões estacionais consistentes, muito marcados. Diferenças apreciáveis na magnitude da CPUE das três espécies-alvo, entre pesqueiros, sugerem diferenças na orientação do esforço de pesca entre pesqueiros, o qual foi, no entanto, difícil de estimar devido ao elevado grau de mistura das três espécies-alvo nas capturas. A diversidade estacional na profundidade dos lances, observadas nalguns pesqueiros (em particular nos mais explorados do Algarve) parecem depender de variações na orientação do esforço, relativamente à espécie-alvo. Foram observadas diferenças consistentes na duração e distância dos lances de arrasto entre pesqueiros, o que parece relacionar-se com as dimensões dos pesqueiros. Contudo, diferenças nas espécies-alvo a que a pesca foi dirigida podem, também, ter afectado a duração dos arrastos.

\section{CONCLUSÕES E DESENVOLVIMENTOS FUTUROS}

Pelo sucesso deste trabalho, conclui-se que a opção pela implementação de um SIG de raíz com recurso à programação é um caminho a considerar em estudos que procurem ensaiar novas metodologias e que lidem com um 
grande volume de informação. O facto de se tratar de uma aplicação stand-alone confere-lhe portabilidade para outros computadores, não exigindo a instalação prévia de qualquer software SIG. É importante, também, pensar-se que um programa como este constitui um património para o futuro, que poderá ser sempre utilizado, e até alterado, para se adaptar a outras necessidades.

Devido ao facto de ser uma aplicação totalmente orientada para os fins científicos do projecto em causa e, portanto, não possuir toda a panóplia de botões e funções que os SIG comerciais apresentam, tem a vantagem de poder ser utilizada por qualquer cientista da área, sem necessitar de treino prévio.

Uma outra opção, no âmbito dos SIG, possível em projectos deste tipo, é a adopção de sistemas open-source, nos quais é possível ter acesso ao código-fonte por trás de cada função e alterá-los à medida das necessidades. Estes sistemas possuem também a vantagem de possuírem numerosas bibliotecas com módulos criados por outros utilizadores, também em open-source, o que permite a construção de SIG orientados para fins específicos e torna o trabalho de programação mais fácil. Um exemplo de um SIG deste tipo é o GRASS (NETELER et al., 2002), que corre no sistema operativo, também open-source, (

Relativamente ao software de BD utilizado neste trabalho, conclui-se que, apesar de ter sido bem sucedido no projecto GEOCRUST, em que foram utilizados dados de apenas dois anos, demonstrou limitações de desempenho, de segurança e mesmo ao nível das funções que providencia. A BD deste projecto encontra-se, possivelmente, perto do limite de tamanho que possibilita a utilização software, sendo aconselhável num estudo semelhante, com maior volume de dados, a utilização de outra aplicação. Uma alternativa no ®WINDows é o ${ }^{\circledR}$ SQL 2000 da ${ }^{\circledR}$ Microsoft e, no ${ }^{\circledR}$ Linux, o ${ }^{\circledR}$ ORACLE ou ${ }^{\circledR}$ PostgresQL, que já deu provas de sucesso em SIG com grande volume de dados ${ }^{7}$.

Para terminar, é de referir o papel importante que as novas tecnologias poderão vir a desempenhar na análise espacial, num futuro próximo. Alguns dos algoritmos utilizados na aplicação GEOCRUST 1.0, nomeadamente o de detecção dos limites dos arrastos, poderão ser melhorados com a utilização de redes neuronais. Esta e outras técnicas de inteligência artificial, como é o caso dos algoritmos genéticos, ou de vida artificial, como é o caso dos autómatos celu$\operatorname{lares}^{8}$, são exemplos de metodologias desenvolvidas no âmbito das ciências da computação que estão a ser utilizadas em estudos de carácter espacial, com recurso a SIG $^{9}$.

Espera-se que o software GEOCRUST 1.0 sirva como percursor de um Sistema Integrado de Aquisição e Análise de Dados de Esforço, Capturas e Rendimentos Geo-referenciados para as Pescarias de Arrasto Portuguesas a desenvolver num próximo projecto. O Sistema deverá ser alargado à pescaria de arrasto de peixe que opera ao largo de toda a costa portuguesa e os vários procedimentos de Marcação de Viagens e, sobretudo, de Marcação de Arrastos deverão ser optimi-

\footnotetext{
7 http://grass.itc.it/sqlgrasslist.html (último acesso em Outubro de 2003).

8 http://www.casa.ucl.ac.uk/agent.htm (último acesso em Outubro de 2003).

9 http://casa.ucl.ac.uk/agent.htm (último acesso em Outubro de 2003).
} 
zados. Neste próximo projecto deverá ser feito um grande esforço no sentido de desenvolver os instrumentos que possam proporcionar, no futuro, a disponibilização selectiva, através da Internet, de informação de cartografia de esforço de pesca e de CPUE para a investigação e gestão pesqueiras mas, também, para a própria indústria (armadores e mestres pescadores).

\section{AGRADECIMENTOS}

Os autores agradecem a colaboração prestada pela Doutora Isabel Afonso-Dias e pela Professora Doutora Cristina Veiga-Pires na preparação, respectivamente, dos resumos em Inglês e em Francês bem como as sugestões de melhoria do documento realizadas por dois revisores anónimos.

\section{BIBLIOGRAFIA}

Afonso-Dias, M.; Simões, J.; Pinto, C. and Sousa, P. (2002) - Use of satellite GPS data to map effort and landings of the portuguese crustacean fleet. Final Report EC-DGXIV/STUDY/99/059.

Neteler, M. and Mitasova, H. (2002) - Open Source GIS: A Grass GIS Approach. Kluwer International Series in Engineering and Computer Science.

VASCONCELOS, M. S. (2000) - The contribution of research to an improvement of fisheries monitoring. Proceedings of the International Conference on Fisheries Monitoring, Control and Surveillance. Brussels, 24-27 September 2000 - http://www.europa.eu.int/comm/fisheries/news_corner/ autres/control/vasconcelos.pdf (último acesso em Janeiro de 2003). 